\title{
Mapping the temperature of the intra-cluster medium with the thermal Sunyaev-Zel'dovich effect
}

\author{
G. Hurier ${ }^{1}$ and C. Tchernin ${ }^{2}$ \\ ${ }^{1}$ Centro de Estudios de Física del Cosmos de Aragón (CEFCA), Plaza de San Juan 1 Planta-2, 44001 Teruel, Spain \\ e-mail: ghurier@cefca.es \\ 2 Center for Astronomy, Institute for Theoretical Astrophysics, Heidelberg University, Philosophenweg 12, 69120 Heidelberg, \\ Germany
}

Received 1 November 2016 / Accepted 11 March 2017

\begin{abstract}
The hot electrons in the intra-cluster medium produce a spectral distortion of the cosmic microwave background (CMB) black body emission, the thermal Sunyaev-Zel'dovich effect (tSZ). This characteristic spectral distortion is now commonly used to detect and characterize the properties of galaxy clusters. The tSZ effect spectral distortion does not depend on the redshift, and is only slightly affected by the galaxy cluster properties via the relativistic corrections, when the electrons reach relativistic velocities. The present work proposes a linear component separation approach to extract the tSZ effect Compton parameter and relativistic corrections for next-generation CMB experiments. We demonstrated that relativistic corrections, if neglected, would induce a significant bias on the galaxy cluster Compton parameter, the tSZ scaling relation slope, as well as tSZ angular power spectrum shape measurements. We showed that $\mathrm{tSZ}$ relativistic correction mapping can be achieved at high signal-to-noise ratio with a low level of contamination up to $\ell=3000$ for next-generation CMB experiments. At smaller angular scales the contamination produced by infrared emission will be a significant source of bias. Such tSZ relativistic correction mapping enables the study of galaxy cluster temperature profile via the tSZ effect only.
\end{abstract}

Key words. galaxies: clusters: general - cosmic background radiation - large-scale structure of Universe

\section{Introduction}

The thermal Sunyaev-Zel'dovich (tSZ, Sunyaev and Zeldovich 1972) is produced by the interaction of cosmic microwave background $(\mathrm{CMB})$ photons with the hot ionized plasma inside galaxy clusters. This effect induces a characteristic spectral distortion of the CMB black body spectral energy distribution (SED).

In the last five years, the measurement of the tSZ effect produced by galaxy clusters has reached an unprecedented accuracy (Marriage et al. 2011; Bleem et al. 2015; Planck Collaboration XXVII 2016).

It is generally assumed that the tSZ spectral distortion is invariant with the galaxy cluster redshift and intra-cluster medium (ICM) physical properties. The redshift invariance of the tSZ effect is satisfied for adiabatic evolution of the Universe and has been confirmed by recent measurements (see e.g., Hurier et al. 2014). However, the invariance with respect to the plasma properties is broken if the electrons of the hot plasma reach relativistic velocities, and relativistic corrections have to be applied (Wright 1979). These relativistic corrections become significant for temperature above a few $\mathrm{keV}$ (electron velocities of $\simeq 0.1 c$ at $5 \mathrm{keV}$ ) and enable the possibility to use the tSZ distortion as a probe to measure the temperature of the hot plasma inside galaxy clusters (Pointecouteau et al. 1998; Ensslin \& Hansen 2004).

The extraction of the tSZ effect is a challenge; the intensity of the effect on the sky is small, around $10^{-6}-10^{-4} K_{\mathrm{CMB}}$, and presents a strong correlation with other astrophysical processes, such as radio-loud active galactic nuclei (AGN) emission, infra-red emission from galaxy cluster member galaxies (Planck Collaboration XXIII 2016). Nevertheless, the construction of tSZ effect Compton parameter maps through component separation is now a mature activity (Hurier et al. 2013; Remazeilles et al. 2011; Bobin et al. 2008), and reaches an angular resolution of 1.4 arcmin (Aghanim et al., in prep.) combining Planck and Atacama Cosmology Telescope (ACT) data. However, the relativistic corrections to the tSZ SED are one order of magnitude smaller than the tSZ effect itself and can be easily confused with other astrophysical processes for experiments that have an unsufficient spectral coverage in the range $[10,1000] \mathrm{GHz}$.

For example, evidence of the $\mathrm{tSZ}$ relativistic corrections have been claimed with Herschel-SPIRE (Zemcov et al. 2010) and ZSpec (Zemcov et al. 2012). Present experiments such as Planck (Planck Collaboration I 2014) have enabled the statistical detection at $5 \sigma$ of the tSZ effect relativistic corrections (Hurier 2017). The future generation of high-resolution high-sensitivity experiments such as $\mathrm{COrE}+{ }^{1}$ will strongly improve the sensitivity and the number of observed frequencies, opening the door to exact measurements of the tSZ relativistic corrections and scientific exploitation of these relativistic corrections.

This paper is dedicated to the reconstruction and mapping of the tSZ relativistic corrections with the MILCA component separation method (Hurier et al. 2013), previously

1 http://hdl.handle.net/11299/169642 
used to reconstruct full sky maps of the tSZ effect (Planck Collaboration XXI 2014; Planck Collaboration XXII 2016). The scientific outcomes of the relativistic correction mapping will be discussed in an upcoming paper (Tchernin \& Hurier, in prep.).

The paper is organized as follows: Sect. 2 describes the tSZ effects and the relativistic corrections to this effect. In Sect. 3 we describe the tSZ relativistic correction map reconstruction methodology and characterize the transfer function of the reconstructed maps. Then, Sect. 4 addresses the drawbacks of neglecting tSZ relativistic corrections for future $\mathrm{CMB}$ experiments. Finally, we discuss the main limitation of the tSZ relativistic corrections recovery in Sect. 5 and we draw our conclusions in Sect. 6

\section{The thermal Sunyaev-Zel'dovich effect}

Through the tSZ effect (Sunyaev \& Zeldovich 1972) CMB photons receive an average energy boost by collision with hot (a few $\mathrm{keV}$ ) ionized electrons of the intra-cluster medium (see e.g., Birkinshaw 1999; Carlstrom et al. 2002, for reviews). The intensity of this effect toward a given line of sight is given by the tSZ Compton parameter,

$y=\int n_{e} \frac{k_{\mathrm{B}} T_{\mathrm{e}}}{m_{\mathrm{e}} c^{2}} \sigma_{\mathrm{T}} \mathrm{d} l$,

where $\mathrm{d} l$ is the distance along the line of sight, and $n_{\mathrm{e}}$ and $T_{\mathrm{e}}$ are the electron number density and temperature, respectively. In units of CMB temperature, the contribution of the tSZ effect for a given observation frequency $v$ is

$\frac{\Delta T_{\mathrm{CMB}}}{T_{\mathrm{CMB}}}=g_{v} y$.

Neglecting relativistic corrections we have

$g_{v} \simeq\left[x \operatorname{coth}\left(\frac{x}{2}\right)-4\right]$,

with $x=h v /\left(k_{\mathrm{B}} T_{\mathrm{CMB}}\right)$. At $z=0$, where $T_{\mathrm{CMB}}(z=0)=2.726 \pm$ $0.001 \mathrm{~K}$, the $\mathrm{tSZ}$ effect is negative below $217 \mathrm{GHz}$ and positive for higher frequencies. This characteristic spectral signature of tSZ makes it a unique tool for the detection of galaxy clusters.

This spectral signature is also slightly dependent on $T_{\mathrm{e}}$ through relativistic corrections (see Nozawa et al. 2000, for a detailed fitting formula). In the present work, the relativistic corrections on the tSZ emission law have been computed as presented in Pointecouteau et al. (1998). Based on this estimation, we assume that the relativistic correction on the tSZ emission law can be described as a first order linear approximation. We note that this assumption is satisfied if the tSZ spectral distortion variations are monotonic with respect to $T_{\mathrm{e}}$,

$\Delta T_{\mathrm{CMB}}^{\mathrm{relat}}\left(T_{\mathrm{e}}\right) \simeq \Delta T_{\mathrm{CMB}}^{\mathrm{unrelat}}+T_{\mathrm{e}} \Delta T_{\mathrm{CMB}}^{\mathrm{cor}}$,

where $\Delta T_{\mathrm{CMB}}^{\text {relat }}\left(T_{\mathrm{e}}\right)$ is the total tSZ induced CMB distortion, $\Delta T_{\mathrm{CMB}}^{\mathrm{unrelat}}$ is the non-relativistic tSZ effect contribution, and $\Delta T_{\mathrm{CMB}}^{\mathrm{COMB}}$ is the relativistic correction per unit of electronic temperature, $T_{\mathrm{e}}$, assuming a first order linear approximation. This further due to the fact that the averaged tSZ emission from the electron populations at various temperatures can be modeled as a single temperature.

This assumption is already implicitly applied when fitting a single temperature to tSZ effect observed on the sky. Indeed, due to the line of sight integration of the tSZ signal, the observed
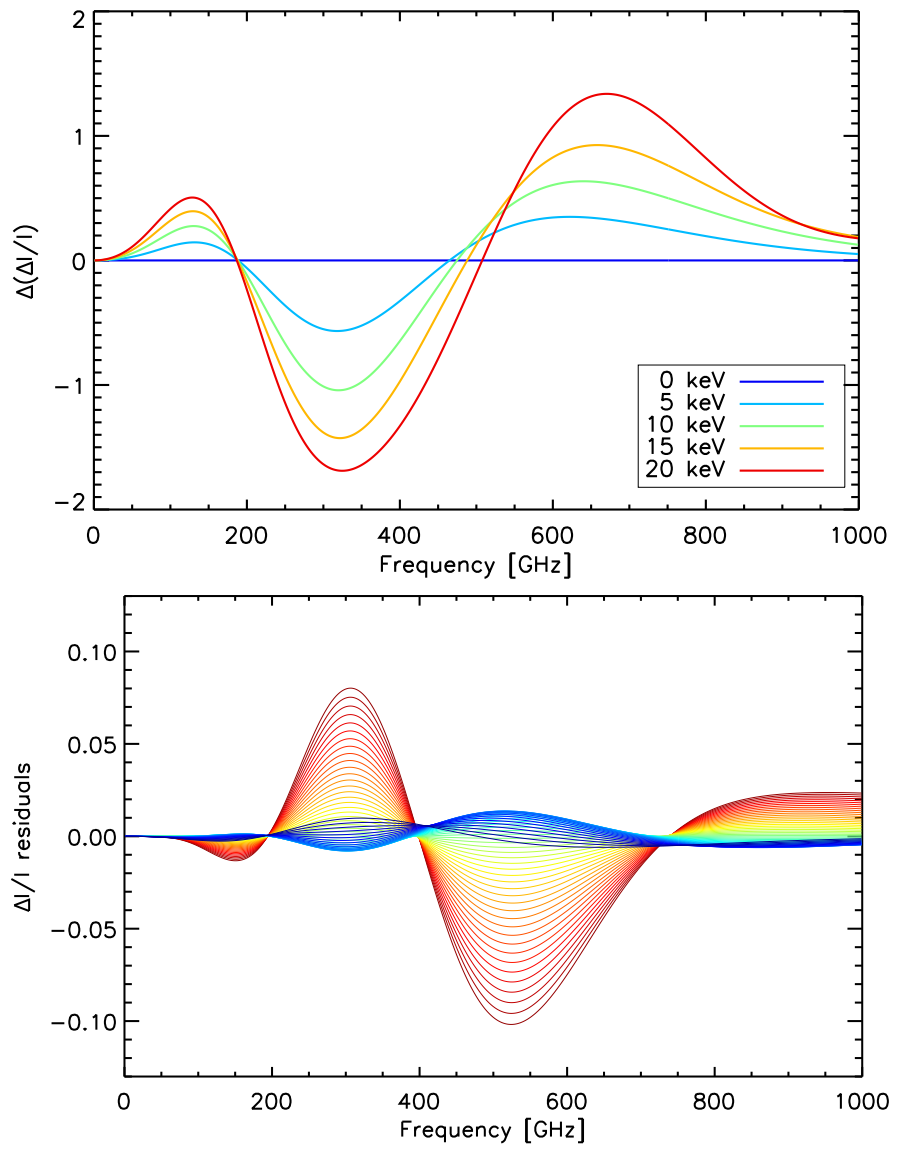

Fig. 1. Top panel: tSZ relativistic corrections to the tSZ spectral distortion as a function of the frequency for various temperatures of the hot plasma from 0 to $20 \mathrm{keV}$. Bottom panel: error on the tSZ spectral distortion induced by the linear approximation from 0 (dark blue solid line) to $10 \mathrm{keV}$ (dark red solid line) as a function of the frequency, using $T_{\mathrm{e}}=5 \mathrm{keV}$ as a reference temperature.

emission is already a linear combination of tSZ effect produced by electrons at different electronic temperatures.

Figure 1 (top panel) shows the relativistic correction additional spectral distortion to the non-relativistic tSZ spectral distortion as a function of the frequency for various temperatures of the hot plasma ranging from 0 to $20 \mathrm{keV}$. Contrary to the nonrelativistic $\mathrm{tSZ}$ effect, $\mathrm{tSZ}$ relativistic corrections are not null at $v \simeq 217 \mathrm{GHz}$, but at $\simeq 188 \mathrm{GHz}$. Consequently, tSZ relativistic corrections induce a shift of the tSZ effect zero frequency, $v_{0}$, that can be parametrized as $v_{0} \simeq 217.4+T_{\mathrm{e}} / 2 \mathrm{GHz}$ with $T_{\mathrm{e}}$ expressed in $\mathrm{keV}$. The relativistic corrections essentially reduce the amplitude of the spectral distortion for frequencies up to $\simeq 450 \mathrm{GHz}$ and increase the $\mathrm{tSZ}$ distortion for higher frequencies. Considering that tSZ effect is usually estimated from low frequency channels (below $500 \mathrm{GHz}$, where CMB emission is significant compared to other emissions on the sky), the tSZ relativistic corrections might induce a bias on measured Compton parameter, which is under-estimated if relativistic corrections are neglected.

We also present in Fig. 1 (bottom panel) the error on the tSZ spectral distortion that is induced by our linear approximation. We show that this error is more than one order of magnitude below the amplitude of the relativistic correction itself. This demonstrates that the linear approximation provides a good description of the tSZ spectral distortion from 0 to $10 \mathrm{keV}$, that is, the typical temperature range of galaxy clusters. We note that in 
a high temperature situation, the reference temperature $T_{\mathrm{e}}$ can be changed to reduce the discrepancy for high-temperature ICM.

\section{Mapping the $\mathrm{tSZ}$ relativistic corrections}

To map the tSZ effect relativistic corrections, we used a component separation approach based on variance minimization (Hurier et al. 2013). This method has been successfully used to recover the tSZ effect Compton parameter with Planck data (Planck Collaboration XXI 2014) and with combined ACT and Planck data (Aghanim et al., in prep.). In this section, we describe the hypothesis made and methodology used to reconstruct the tSZ effect relativistic corrections, and we carefully study the key frequencies for foreground/background emissions removal.

\subsection{MILCA approach}

We assumed that the sky can be well described as a linear mixture of astrophysical sources,

$\mathbf{T}=\mathcal{A} \mathbf{S}+\mathbf{N}$,

where $\mathbf{T}$ are the observed frequency channels, $\mathcal{A}$ is the mixing matrix, $\mathbf{S}$ are the astrophysical components and $\mathbf{N}$ is the instrumental noise. The MILCA method is, by construction, a linear method aiming at extracting a given component, knowing its spectral behavior, from multi-channel observations of the sky. However, the relativistic corrections to the tSZ effect are nonlinear.

Consequently, to apply the MILCA approach to the tSZ relativistic corrections reconstruction, we first performed a linear approximation of $\mathrm{tSZ}$ relativistic correction SED. We modeled the tSZ spectral distortion, $g_{v, T_{\mathrm{e}}}$, as follows

$g_{v, T_{\mathrm{e}}} \simeq g_{v, 0}+T_{\mathrm{e}} h_{v}$,

where $g_{v, T_{\mathrm{e}}}$ is the tSZ spectral distortion presented in Sect. 2 at the temperature, $T_{\mathrm{e}}$, of the hot electrons in the intra-cluster medium, and $h_{v}$ is a linear approximation of the $\mathrm{tSZ}$ relativistic corrections.

In the present analysis we computed $h_{v}$ as

$h_{v}=\frac{g_{v, T_{1}}-g_{v, 0}}{T_{1}}$,

for $T_{1}=5 \mathrm{keV}$, considering that the typical temperature of galaxy clusters is a few $\mathrm{keV}$. To increase the accuracy of the reconstruction method, $T_{1}$ can be set to any temperature depending on the concerned galaxy cluster average temperature.

The MILCA method achieves component separation through a minimization of the variance of the reconstructed map under constraints. It allows us to perform a localized reconstruction of the astrophysical component both in real and Fourrier spaces.

The most simple estimator of tSZ relativistic corrections reads

$S_{r}=\left(\mathbf{f}_{v}^{\mathrm{T}} C_{\mathbf{T}}^{-1} \mathbf{f}_{v}\right)^{-1} \mathbf{f}_{v}^{\mathrm{T}} C_{\mathbf{T}}^{-1} \mathbf{T}$,

with $S_{r}$ the relativistic corrections map, $\mathbf{f}_{v}^{\mathrm{T}}$ it's frequency dependence, and $C_{\mathbf{T}}^{-1}$ the covariance matrix of the frequency channel maps $\mathbf{T}$. This estimator is a standard linear fit that minimizes the variance of the reconstructed signal.

However, it is well-known that variance minimisation is biased by spatial correlations between the component being extracted and other astrophysical components (Hurier et al. 2014).
In the present case, the Compton parameter, $y$, and the relativistic correction signal $r$ are, by construction, highly spatially correlated. Consequently, we need to avoid bias on the $r$-map by adding an extra constraint ${ }^{2}$ that removes the contribution from non-relativistic tSZ effect. The estimator becomes

$\mathbf{S}=\left(\mathcal{F}^{\mathrm{T}} C_{\mathbf{T}}^{-1} \mathcal{F}\right)^{-1} \mathcal{F}_{v}^{\mathrm{T}} C_{\mathbf{T}}^{-1} \mathbf{T}$,

where $\mathcal{F}$ is a rectangular matrix that contains the spectral behavior of the $n_{c}$ components on which a constraint is applied, and $\mathbf{S}$ is a vector that contains the $n_{c}$ maps corresponding to the constrained astrophysical components. We note that $S_{r}=\mathbf{e}_{r}^{\mathrm{T}} \mathbf{S}$, with $\mathbf{e}_{r}$ a vector that selects the subspace corresponding to the tSZ relativistic corrections component.

Additionally, the frequency maps covariance matrix, $C_{\mathbf{T}}$, is not known and has to be estimated from the data. This matrix can be decomposed as,

$C_{\mathrm{T}}=\mathcal{A}^{\mathrm{T}} C_{\mathrm{S}} \mathcal{A}+C_{\mathbf{N}}$.

The astrophysical component covariance matrix $C_{\mathbf{S}}$ and the instrumental noise covariance matrix $C_{\mathbf{N}}$ do not have the same eigenvectors. Consequently, $\mathcal{C}_{\mathbf{N}}$ adds non-physical correlations to $C_{\mathbf{S}}$. Similarly to physical correlations, these correlations will add some bias to the variance minimisation process. Consequently, we apply the following transformation,

$\tilde{C}_{\mathbf{T}}=C_{\mathbf{T}}-C_{\mathbf{N}}-\mathcal{F}\left(\mathcal{F}^{\mathrm{T}} C_{\mathbf{T}}^{-1} \mathcal{F}\right)^{-1} \mathcal{F}^{\mathrm{T}}$,

to remove noise-induced bias and suppress the contribution from constrained components to the covariance matrix. Then, we performed a rank reduction on $\tilde{C}_{\mathbf{T}}$, keeping only the highest eigenvalues corresponding to the number of unconstrained astrophysical components to minimize. Other eigenvalues are constrained by minimizing the noise contribution in the reconstructed map

$V_{\mathbf{N}, r}=\mathbf{e}_{r}^{\mathrm{T}} \boldsymbol{W}_{\mathrm{r}}^{\mathrm{T}} \mathcal{C}_{\mathbf{N}} \boldsymbol{W}_{\mathrm{r}} \mathbf{e}_{r}$

with $\mathcal{W}_{\mathrm{r}}^{\mathrm{T}}=\left(\mathcal{F}^{\mathrm{T}} \tilde{\mathcal{C}}_{\mathrm{T}}^{-1} \mathcal{F}\right)^{-1} \mathcal{F}_{v}^{\mathrm{T}} \tilde{C}_{\mathrm{T}}^{-1}$. We remind that the minimization is performed on the eigenvalues suppressed by the rank reduction of $\tilde{C}_{\mathrm{T}}$.

Finally the tSZ relativistic correction map is given by

$S_{\mathrm{r}}=\mathbf{e}_{r}^{\mathrm{T}} \mathcal{W}_{\mathrm{r}}^{\mathrm{T}} \mathbf{T}$.

Similarly, the Compton parameter map is obtained by minimizing

$V_{\mathbf{N}, y}=\mathbf{e}_{y}^{\mathrm{T}} \boldsymbol{W}_{\mathrm{y}}^{\mathrm{T}} C_{\mathbf{N}} \boldsymbol{W}_{\mathrm{y}} \mathbf{e}_{y}$,

with $\mathbf{e}_{y}$ a vector that selects the subspace corresponding to the tSZ Compton parameter map. The $y$-map is computed as

$S_{\mathrm{y}}=\mathbf{e}_{y}^{\mathrm{T}} \boldsymbol{W}_{\mathrm{y}}^{\mathrm{T}} \mathbf{T}$.

This approach allows us to build a tSZ Compton parameter map $y=\frac{k_{\mathrm{B}} \sigma_{\mathrm{T}}}{m_{\mathrm{e}} c^{2}} \int n_{\mathrm{e}} T_{\mathrm{e}} \mathrm{d} l$, and a map of $\mathrm{tSZ}$ relativistic corrections $r \simeq$ $\frac{k_{\mathrm{B}} \sigma_{\mathrm{T}}}{m_{\mathrm{e}} c^{2}} \int n_{\mathrm{e}} T_{\mathrm{e}}^{2} \mathrm{~d} l$. This dependency can be derived from Eq. (7). The $\mathrm{tSZ}$ distortion at a given frequency is given by $\Delta T / T \simeq g_{v, 0} y+$ $h_{v} r$.

A more detailed description of the MILCA method can be found in Hurier et al. (2013).

\footnotetext{
2 Extra-constraints can be added for any astrophysical component that presents a well known spectral behavior, which is the case, for example, of non-relativistic tSZ effect and CMB emission.
} 

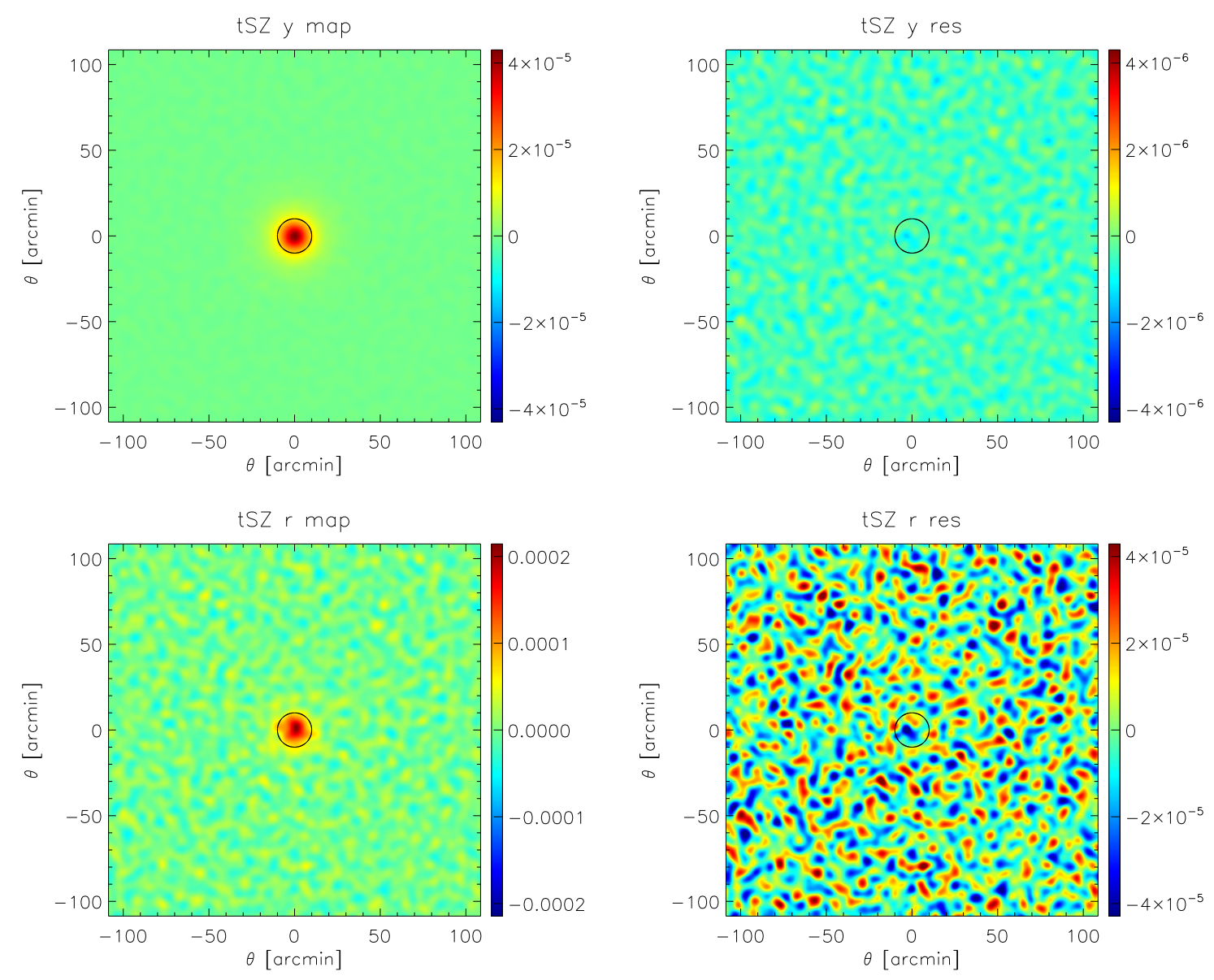

Fig. 2. From left to right and top to bottom: reconstructed tSZ $y$-map, residuals in the reconstructed $y$-map, reconstructed tSZ $r$-map, residuals in the reconstructed $r$-map for a patch of $3.5 \times 3.5$ degrees, with 0.4 arcmin pixels, centered on a simulated galaxy cluster at 7 arcmin FWHM angular resolution.

\subsection{Test on simulated datasets}

We are now interested in testing the algorithm described above. Thus, we have constructed simulations of the full microwave and submillimeter sky as it will be observed by next generation CMB experiments like COrE+ (a summary of the planned characteristic can be found in Table 1) using a set of template maps to reproduce astrophysical components. A complete description of the simulation procedure can be found in Hurier et al. (2013). Within the considered components, we include CMB, diffuse Galactic emissions (synchrotron, free-free and thermal dust), Galactic CO emission, and Galactic and extragalactic point sources. We also added the cosmic infrared background contribution following the best fitting model from Planck Collaboration XXIII (2016).

Each template is assumed to be the real sky signal and is convolved by the point spread function of each COrE+ channel, and the component SEDs are convolved by the corresponding bandpass assuming $\Delta v / v=0.2$. Finally, we added a homogeneous instrumental noise according to the COrE+ detector sensitivity for the 21 observed frequencies. We note that MILCA weights are constructed to be insensitive to the addition or the substraction of tSZ signal in the input maps, under the assumtion that the tSZ signal is not spatially correlated with other astrophysical components (Eq. (11)). Consequently, the MILCA weights can be estimated without any tSZ emission on the sky. We stress that these simulations assume a single SED for each component (except for the CIB). We will discuss in the following the impact of varying SED on the sky.

We simulated a galaxy cluster following a universal pressure profile (Arnaud et al. 2010; Planck Collaboration Int. V 2013), $P_{\mathrm{e}}$, and a polytropic relation between temperature, $T_{\mathrm{e}}$, and density, $n_{\mathrm{e}}$, with a polytropic index $\delta=1.2 \mathrm{such}$ as $P_{\mathrm{e}}=n_{\mathrm{e}} T_{\mathrm{e}}=n_{\mathrm{e}}^{\delta}$. We divided the clusters into cells of $0.05 R_{500}$, computed the tSZ effect produced by each cell individually accordingly to the cell densities and temperatures, and applied the corresponding spectral distortion to the COrE+ simulated frequency channels by projecting the galaxy cluster tSZ effect on the line-of-sight. We stress that, due to relativistic corrections, the tSZ effect cannot be described with a single spectral distortion and a Compton parameter map.

Under the approximation we are doing for the MILCA-based component separation, we should recover a map of the integrated pressure on the line of sight and a map of the integrated product of pressure and temperature on the line of sight.

Figure 2 presents the reconstructed tSZ $y$ and $r$-maps at 7 arcmin FWHM resolution for a nearby massive galaxy cluster $\left(M_{500}=8 \times 10^{14} M_{\odot}, z=0.06\right)$. This reconstruction is presented as an example and a proof of concept. In the following we rely on analytical estimations of the noise and residuals in the MILCA tSZ maps. At this resolution the noise level in MILCA maps is $2.74 \times 10^{-7}$ in the $y$-map and $1.53 \times 10^{-5} \mathrm{keV}$ in the $r$-map. For the considered galaxy cluster, the $y$-signal peaks at $y=7.1 \times 10^{-5}$. Thus, the noise level in the $r$-map can be converted to a noise level on the temperature at the peak $\Delta T_{\mathrm{e}}=0.2 \mathrm{keV}$. However, 
G. Hurier and C. Tchernin: Mapping the temperature of the intra-cluster medium with the thermal Sunyaev-Zel'dovich effect
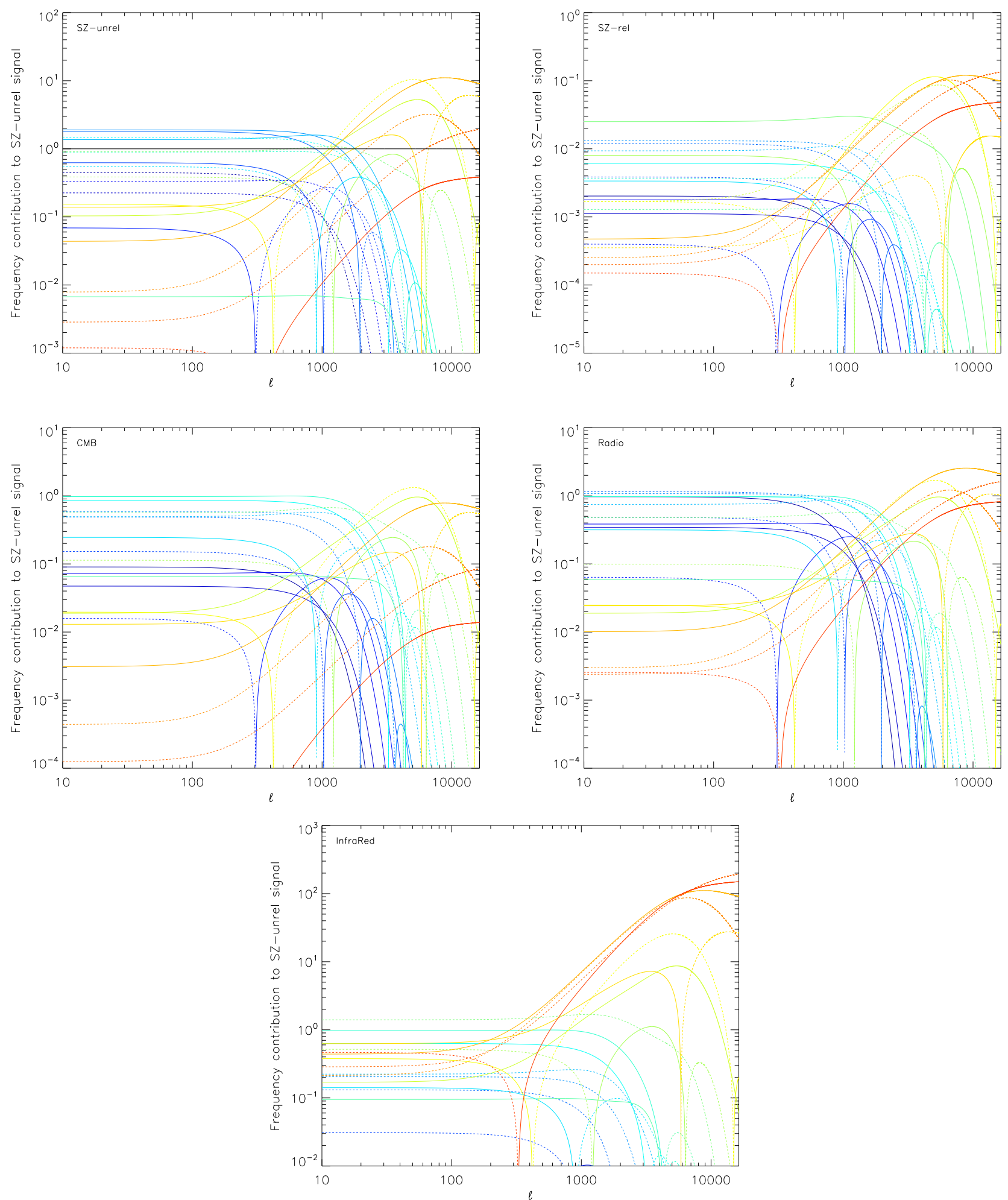

Fig. 3. Contributions received by the tSZ Compton parameter map as a function of the angular scale. From left to right and top to bottom: contribution of each frequency (from $30 \mathrm{GHz}$ in dark blue to $800 \mathrm{GHz}$ in red for the 21 frequencies of COrE+) to non-relativistic tSZ effect, relativistic corrections to tSZ effect, $\mathrm{CMB}$, Radio component, and infrared components in the reconstructed map. Solid lines indicate positive contributions and dashed lines negative contributions.

we note that this uncertainty will strongly increase in the outskirts of the galaxy cluster due to a fainter $y$ signal.

This result demonstrates that a COrE+ like experiment will achieve a noise level in Compton parameter maps ten times lower than the Planck experiment at the same angular scale. We verified that using the same simulations for Planck data, we recover a noise level consistent with the public Planck tSZ maps.

We observe that the signal in the $r$-map is less extended than the signal in the $y$-map. Indeed the $r$-map is sensitive to the product $T_{\mathrm{e}}^{2} n_{\mathrm{e}}$ and our simulation assumes a polytropic profile. Thus, 
Table 1. Main characteristics (frequencies, beams, number of detectors $N_{\text {det }}$, and sensitivity) planned for the COrE+ experiment.

\begin{tabular}{cccc}
\hline \hline Channel [GHz] & Beam [arcmin] & $N_{\text {det }}$ & $\Delta T[\mu \mathrm{K}$ arcmin] \\
\hline 60 & 14 & 28 & 9.8 \\
70 & 12 & 30 & 9.1 \\
80 & 10.5 & 64 & 6.1 \\
90 & 9.33 & 102 & 4.8 \\
100 & 8.4 & 120 & 4.3 \\
115 & 7.3 & 196 & 3.4 \\
130 & 6.46 & 264 & 3.0 \\
145 & 5.79 & 388 & 2.5 \\
160 & 5.25 & 534 & 2.3 \\
175 & 4.8 & 554 & 2.4 \\
195 & 4.31 & 600 & 2.5 \\
220 & 3.82 & 490 & 3.2 \\
255 & 3.29 & 486 & 4.1 \\
295 & 2.85 & 260 & 8.1 \\
340 & 2.45 & 200 & 14.6 \\
390 & 2.15 & 120 & 33.7 \\
450 & 1.87 & 120 & 71.4 \\
520 & 1.62 & 120 & 181.1 \\
600 & 1.4 & 120 & 551 \\
700 & 1.2 & 60 & 3293.5 \\
800 & 1.05 & 60 & 14499.8 \\
\hline
\end{tabular}

Notes. For each channel the bandwidth is $\Delta v / v=0.25$.

we expect to observe a more compact emission in the $r$-map than in the $y$-map. We did not observe significant bias in the residuals of the $y$-map and the $r$-map, which indicates that systematic effects produced by our linear approximation are not significant compared to the statistical noise level.

\subsection{Frequency contributions}

The MILCA method performs a localized component separation both in real and Fourrier spaces. The COrE+ setting involves different angular resolutions for the different frequency channels. Consequently, the optimal weights for the reconstruction intrinsically depend on the considered angular scale, $\ell$.

The transfer function of a given component, $c$, as a function of scale, $\ell$, reads

$\mathcal{T}_{c}(\ell)=\mathbf{e}_{c}^{\mathrm{T}} \mathcal{W}_{\mathrm{y}}^{\mathrm{T}}(\ell) \mathcal{F} \mathbf{e}_{c}$.

Similarly, the contribution of the component, $i$, to the recovered map for the component $c$ reads

$\mathcal{T}_{i, c}(\ell)=\mathbf{e}_{c}^{\mathrm{T}} \mathcal{W}_{\mathrm{y}}^{\mathrm{T}}(\ell) \mathbf{F}_{i}$,

where $\mathbf{F}_{i}$ is the SED of the component $i$. The contributions of the frequency $v$ to these transfer function are

$$
\begin{gathered}
\mathcal{T}_{c}(\ell, v)=\left[\mathbf{e}_{c}^{\mathrm{T}} \mathcal{W}_{\mathrm{y}}^{\mathrm{T}}(\ell) \mathbf{e}_{v}\right]\left[\mathbf{e}_{v}^{\mathrm{T}} \mathcal{F} \mathbf{e}_{c}\right], \\
\mathcal{T}_{i, c}(\ell, v)=\left[\mathbf{e}_{c}^{\mathrm{T}} \mathcal{W}_{\mathrm{y}}^{\mathrm{T}}(\ell) \mathbf{e}_{v}\right]\left[\mathbf{e}_{v}^{\mathrm{T}} \mathbf{F}_{i}\right],
\end{gathered}
$$

with $\mathbf{e}_{v}$ a vector that selects the subspace corresponding to the frequency $v$. We note that $\mathbf{F}_{i}^{T} \mathbf{e}_{v}$ is a scalar quantity corresponding to the transmission of the component $i$ into the frequency channel $v$.

In order to determine the key frequency channels, we tracked the contributions of the 21 frequencies of COrE + in the $y$-map and $r$-map reconstruction process. These contributions are estimated analytically using Eq. (18), assuming a reference spectral
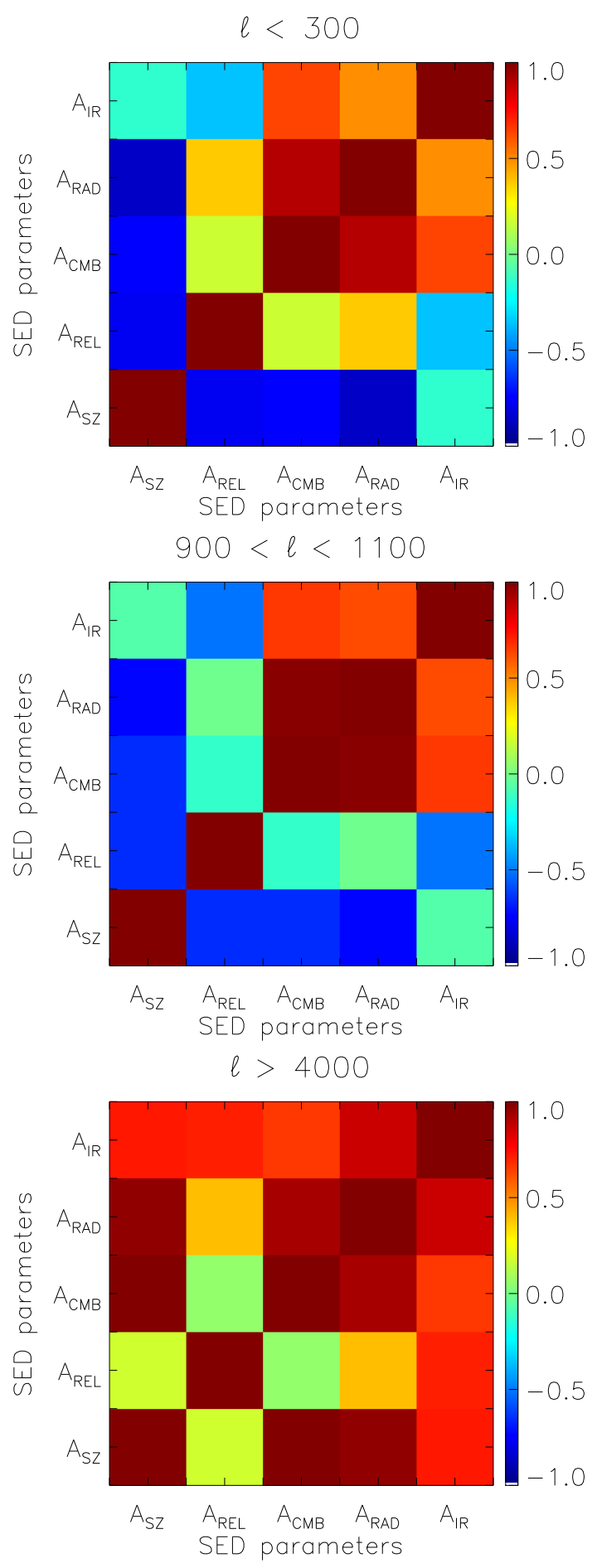

Fig. 5. From top to bottom: correlation matrices between the main astrophysical components for the reconstruction of tSZ $y$-map and $r$-map, at low- $\ell(\ell<300)$, mid- $\ell(\ell \simeq 1000)$, and high- $\ell(\ell>4000)$.

behavior for each astrophysical component. The linear combination weights, $\mathcal{W}$, are computed from the full sky simulations presented in Sect. 3.2.

\subsubsection{MILCA thermal Sunyaev-Zel'dovich $y$-map}

Figure 3 presents the contribution of each frequency to the recovered tSZ Compton parameter map. We also present the 
G. Hurier and C. Tchernin: Mapping the temperature of the intra-cluster medium with the thermal Sunyaev-Zel'dovich effect
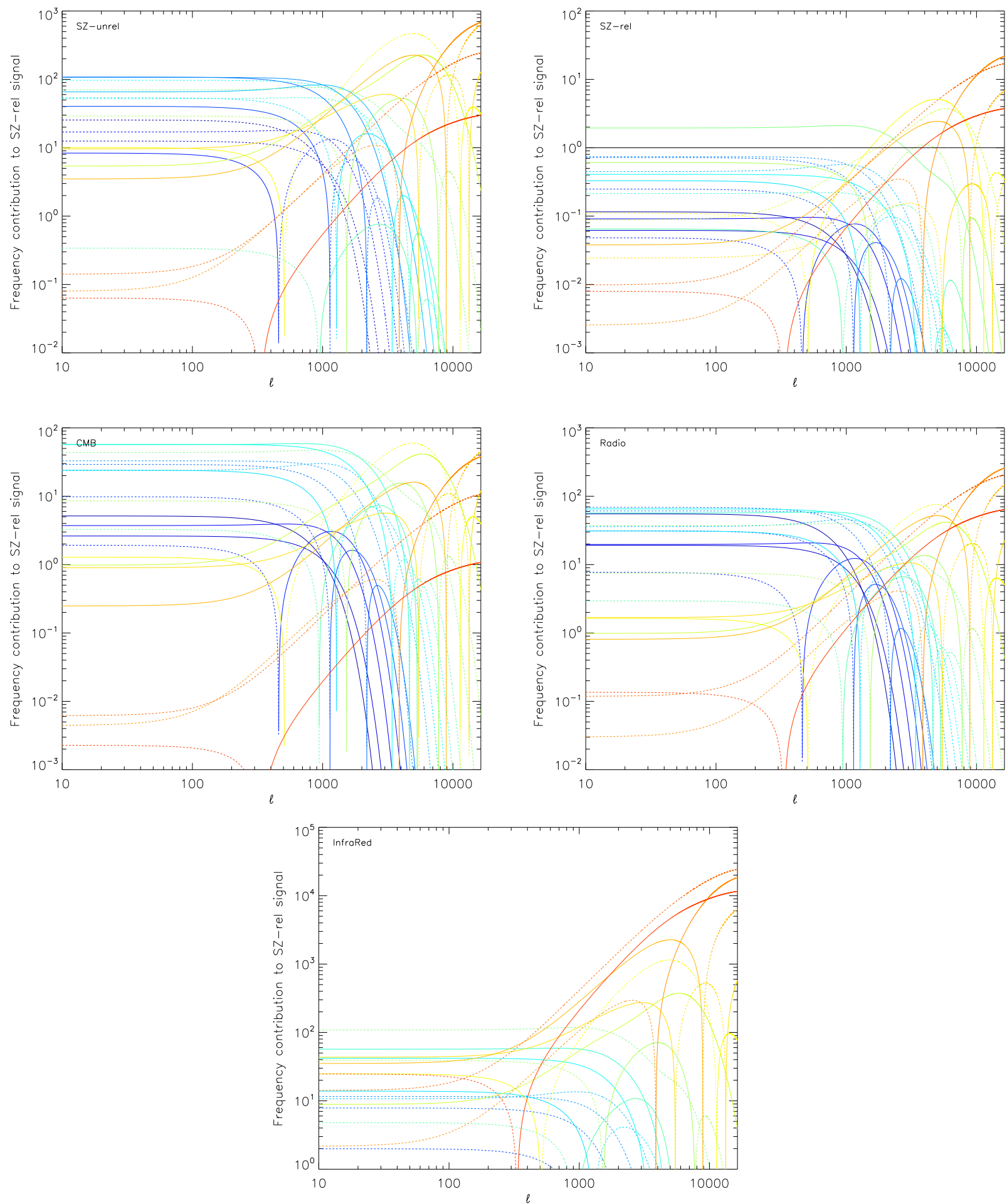

Fig. 4. Contributions received by the tSZ relativistic corrections map as a function of the angular scale. From left to right and top to bottom: contribution of each frequency (from $30 \mathrm{GHz}$ in dark blue to $800 \mathrm{GHz}$ in red for the 21 frequencies of COrE+) to non-relativistic tSZ effect, relativistic correction to tSZ effect, CMB, Radio component, and infrared components of the reconstructed map. Solid lines indicate positive contributions and dashed lines negative contributions.

contribution of each frequency to the suppression of tSZ relativistic corrections, $\mathrm{CMB}$, Radio, and infrared components.

By construction, the sum of all the curves in the tSZ Compton parameter panels is 1 , for all other panels the summation of all curves is 0 .

We observe that at large angular scales the recovery of the tSZ $y$-map is driven by the lowest frequencies up to $255 \mathrm{GHz}$ (shown in blue and green colors on Fig. 3). For $\ell>2000$, the reconstruction is dominated by the highest frequency channel that presents the highest angular resolution. For this small angular scale, we observe a general increase, by a factor of 5 , of the weights for the linear combination. This effect is particularly enhanced for the infrared component cleaning (bottom panel). Such an increase of the weights also implies an increase of the 
noise level and an increase in the leakage from other astrophysical components.

\subsubsection{MILCA thermal Sunyaev-Zel'dovich $r$-map}

Figure 4 presents the same information as Fig. 3 but for the tSZ relativistic corrections map. Consequently, the sum of all the curves in the tSZ relativistic corrections panels average to 1 , and to 0 for all other panels.

We observe that the tSZ relativistic correction reconstruction is also dominated by the $255 \mathrm{GHz}$ channel up to $\ell=2000$. At larger angular scales, radio emission and CMB cleaning is performed using the lowest-frequency channels. At higher angular resolution, lowest frequency channels are no longer available, and highest frequency channels are used, similarly to what we saw for the $y$-map, to reconstruct the tSZ $r$-map and especially clean for infrared contamination.

\subsubsection{Component noise covariance matrix}

The correlation in the noise of the recovered components is very informative on the potential leakage of a given component into another one. If we assume that all components are completely removed by the linear combination, the component noise covariance matrix, $\mathcal{V}_{\mathbf{N}}$, can be computed as

$\mathcal{V}_{\mathbf{N}}=\left(\mathcal{F}^{\mathrm{T}} C_{\mathbf{N}}^{-1} \mathcal{F}\right)^{-1}$

This covariance matrix depends only on the astrophysical component SED properties, $\mathcal{F}$, and on the instrument frequency channel noise covariance matrix, $C_{\mathbf{N}}^{-1}$. Considering that the resolution of each observed channel varies, and assuming that the noise in each frequency band is not spatially correlated, $C_{\mathbf{N}}^{-1}$ varies with the angular scale. In Fig. 5, we present the noise correlation matrices for:

- tSZ Compton paramter, $A_{\mathrm{SZ}}$;

- tSZ relativistic corrections, $A_{\mathrm{REL}}$;

- CMB, $A_{\mathrm{CMB}}$;

- a radio component, $A_{\mathrm{RAD}}$;

- an infra-red component, $A_{\mathrm{IR}}$;

at three different angular scales: $\ell<300,900<\ell<1100$, and $\ell>4000$. For low and mid-angular resolution correlation matrices, we observed a high level of anti-correlation between tSZ $y$ map and $r$-map. This is induced by the strong contribution of frequencies below $400 \mathrm{GHz}$ where $y$-map and $r$-map have opposite signs for their contribution to the intensity maps. Similarly, the $y$-map noise is anti-correlated with the $\mathrm{CMB}$ noise considering that the reconstruction is dominated by low-frequency channels. This behavior changes at high angular resolution, where we observed a significant level of correlation. The increase of the correlation level for high angular resolution shows that the number of frequencies available for high angular resolution starts to be too small and induces a more complex separation of each component. In general, we observe that the reconstruction of the tSZ relativistic corrections is essentially limited by the nonrelativistic $\mathrm{tSZ}$ effect and by the infrared emission that mimics the high-frequency part of the tSZ relativistic correction SED.

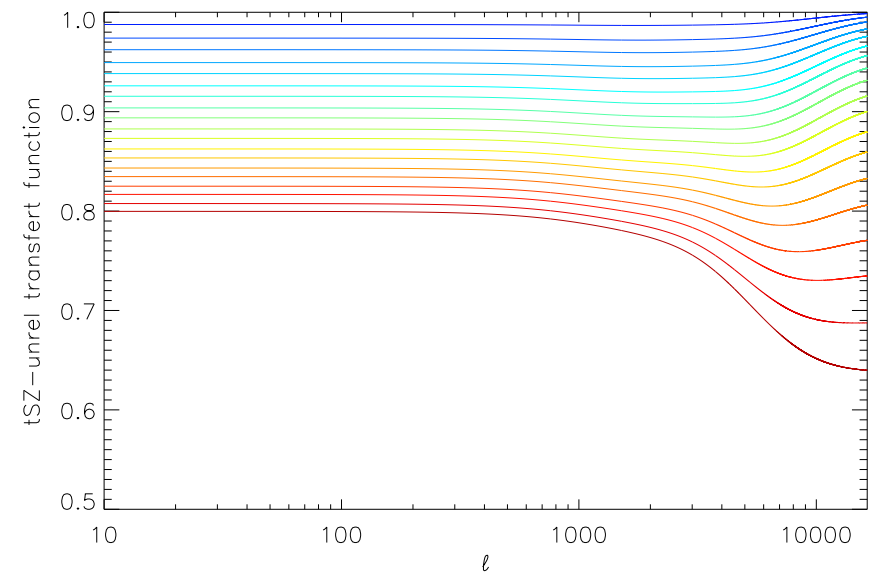

Fig. 6. Transfer function of the tSZ effect as a function of the scale $\ell$ and the temperature $T_{\mathrm{e}}$, from 0 (dark blue) to $15 \mathrm{keV}$ (dark red), when neglecting the relativistic corrections during the reconstruction process for a COrE+.

\section{Impact of relativistic corrections on Compton-parameter-focused analyses}

In this section, we determine the importance of considering tSZ relativistic corrections for Compton-parameter-focused analyses.

\subsection{Component separation transfer function}

We applied Eq. (17) to compute the transfer function of the real tSZ spectral distortion, $g_{v, T_{\mathrm{e}}}$ through a linear component separation that neglects relativistic corrections and only accounts for the non-relativistic tSZ spectral distortion. Figure 6 shows the transfer function of the Compton parameter as a function of the temperature of the ICM and the considered angular scale. For low temperatures, we observe a bias consistent with zero. However at high temperature, we observe a significant bias of the tSZ flux of the cluster that reaches $20 \%$ for $T_{\mathrm{e}}=15 \mathrm{keV}$. We also observe changes in the behavior of the bias at high- $\ell$ due the change of dominating frequencies in the reconstruction. This bias only affects the overall normalization and induces an underestimation of the Compton parameter at high temperatures. The high angular-relation variation of the transfer function may also produce some distortions of the tSZ profiles in the core of galaxy clusters. This underestimation of the tSZ flux when neglecting tSZ relativistic correction can be easily understood from the SED of non-relativistic tSZ effect and tSZ relativistic corrections that reduce the amplitude of the tSZ spectral distortion for frequencies below $400 \mathrm{GHz}$. We stress that this bias is strongly dependent on the noise covariance matrix, as seen by the scale dependence of the bias at high- $\ell$ in Fig. 6. Consequently, this bias is also strongly dependent on the setting used for the experiment. For example, in the case of the Planck experiment, we derived that the induced bias is about a factor of two smaller than for a COrE+ like experiment.

We note that the galaxy cluster tSZ fluxes in the context of the Planck mission have been calibrated without considering the relativistic corrections. Thus, these tSZ fluxes are consistent with the fluxes in the Planck tSZ $y$-map. This has been shown in Planck Collaboration XXII (2016) over a large range of $\ell$ from 100 to 1000 . Consequently, cosmological constraints extracted using the $Y-M$ scaling relation calibrated on the Planck data 


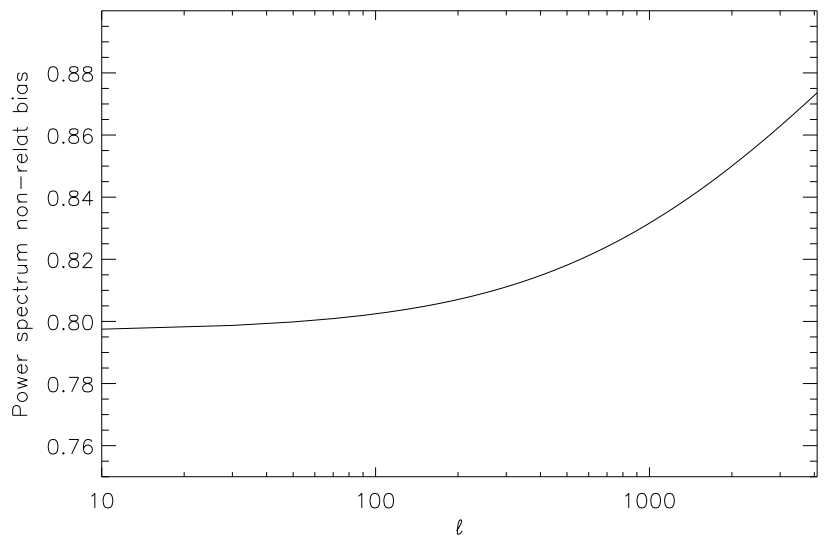

Fig. 7. tSZ auto-correlation angular power spectrum distortion induced by relativistic corrections to the component-separated tSZ $y$-map.

(Planck Collaboration XXIX 2014; Planck Collaboration XXII 2016) are not affected by this bias, as all the bias cancels due to scaling relations being calibrated on the same data.

However, this challenges the meaning of using hydrodynamic-simulations (see e.g., Horowitz \& Seljak 2017) to constrain cosmological parameters. Indeed, the tSZ intensity in the Planck tSZ $y$-map, that neglects relativistic corrections (Planck Collaboration XXII 2016), is biased (up to $20 \%$ at $15 \mathrm{keV}$ ) compared to the total amount of pressure in galaxy clusters.

\subsection{Scaling relation and power spectrum distortion}

We have shown in Sect. 4.1 that tSZ fluxes are biased when the relativistic corrections to the $\mathrm{tSZ}$ spectral distortion are neglected. Given that the amplitude of this bias is related to the temperature of the ICM, this bias also induces distortions on the mass-observable relation, $Y-M$, and on the $\mathrm{tSZ}$ power spectrum shape.

When neglected, relativistic corrections to tSZ effect produce an under-estimation of the high-temperature galaxy cluster Compton parameter that leads to a lower slope for the $Y-M$ relation. For scaling relation, this bias depends on the considered mass range, redshift range, and experiment. For a COrE + like experiment, this bias would be $\simeq-0.05$ on the scaling relation slope, for galaxy cluster masses between $10^{14}$ and $10^{15} M_{\odot}$ and redshift below 0.5 . For the Planck experiment, we estimated a bias of $\simeq-0.03$ on the $Y-M$ slope.

Similarly to Planck Collaboration XXII (2016) and Hurier et al. (2015), we used a halo model to predict the tSZ angular power and characterize the impact of relativistic corrections of the tSZ angular power spectrum. On Fig. 7, we present the ratio between the model that accounts for the relativistic correctioninduced bias, and the model neglecting relativistic corrections for the same $Y-M$ mass-observable relation. We observe that at low $\ell$ the power spectrum is dominated by objects that present a $10 \%$ bias (corresponding to $\simeq 20 \%$ bias on the tSZ power spectrum) on the Compton parameter flux when neglecting relativistic corrections. We stress that the overall normalization of this figure is not relevant due to $Y-M$ calibration processing on the data. However, the distortion is clearly observed, as a consequence of a modification of the slope of the $Y-M$ relation. Consequently, accounting for relativistic corrections of the tSZ spectral distortion is not optional for future experiments, but is required for precision astrophysics that can be done with the tSZ effect, and is mandatory to perform relevant comparisons with numerical simulations.

\section{Systematic effects on reconstructed relativistic corrections map}

We have shown that MILCA allows us to recover the tSZ signal and $\mathrm{tSZ}$ relativistic corrections at high-S/N assuming the sensitivity of the COrE + experiment, improving the sensitivity of Planck to the tSZ effect by a factor of 10 .

\subsection{Nonlinearity}

The main systematic effect comes from our linear approximation of the tSZ relativistic correction approximation. Indeed, the amplitude of relativistic corrections are not exactly linearly related to the temperature of the ICM. To estimate the component mixing and the effective transfer fonction of the $r$-map, we applied Eq. (17) to compute the transfer function of the real tSZ spectral distortion, $g_{v, T_{\mathrm{e}}}$ to our reconstruction of the tSZ $y$-map and $r$ map. We note that the non-relativistic tSZ effect is a well known spectrum that does not vary from cluster to cluster, thus the related transfer function is equal to $\simeq 1$ and there is no leakage into other component maps at the calibration incertitude level of the experiment concerned. A similar argument applies for the CMB component.

In Fig. 8, we present the amplitude of the leakage of tSZ relativistic corrections into the non-relativistic tSZ $y$-map, and the transfer function of the tSZ relativistic corrections to the tSZ $r$ map as a function of the ICM temperature. We observe that the tSZ relativistic correction does not introduce a significant bias into the tSZ $y$-map. The amplitude of relativistic correction is $y T_{\mathrm{e}}$. Even at $10 \mathrm{keV}$, at low- $\ell$ only $0.2 \%$ of the relativistic correction contaminates the $y$-map, implying a bias of $2 \%$ in the tSZ $y$-map. This leakage must be compared with the $20 \%$ bias introduced when neglecting the relativistic corrections as shown by Figs. 6 and 7. The leakage strongly increases at higher $\ell$, where the separation between non-relativistic and relativistic tSZ contributions is harder to achieve, leading to higher value for the weights of the linear combination, and thus to a higher level of bias in the case of a SED mismatch. We also observe that the transfer function for relativistic corrections amplitude in the $r$ map is almost flat up to $\ell=2000$. Thus this effect can be seen as a bias on the overall normalization; the bias is monotonically related to the temperature, that allows to correct the galaxy clusters flux in the $r$-map once the flux in the $y$-map is known. This is generally the case as the noise in the $y$-map is one order of magnitude below the noise in the $r$-map. At first order, the bias can be corrected as follows

$\tilde{r}=(1+\alpha) r$

with $\alpha$ an experiment-dependent correction factor that accounts for the nonlinearity of the $\mathrm{tSZ}$ relativistic correction spectral distortion with respect to the temperature of the ICM. In the present case, $\alpha \simeq 0.04\left(\frac{r}{y}-T_{1}\right)$. This nonlinear correction factor depends on $r / y$ ratio. However, considering that pixels with a significant signal for $r$ will, by construction, have a high $\mathrm{S} / \mathrm{N}$ ratio measurement of $y$. Consequently, the $r / y$ ratio nonlinear correction is not a limitation for the scientific exploitation of the tSZ relativistic corrections mapping. 

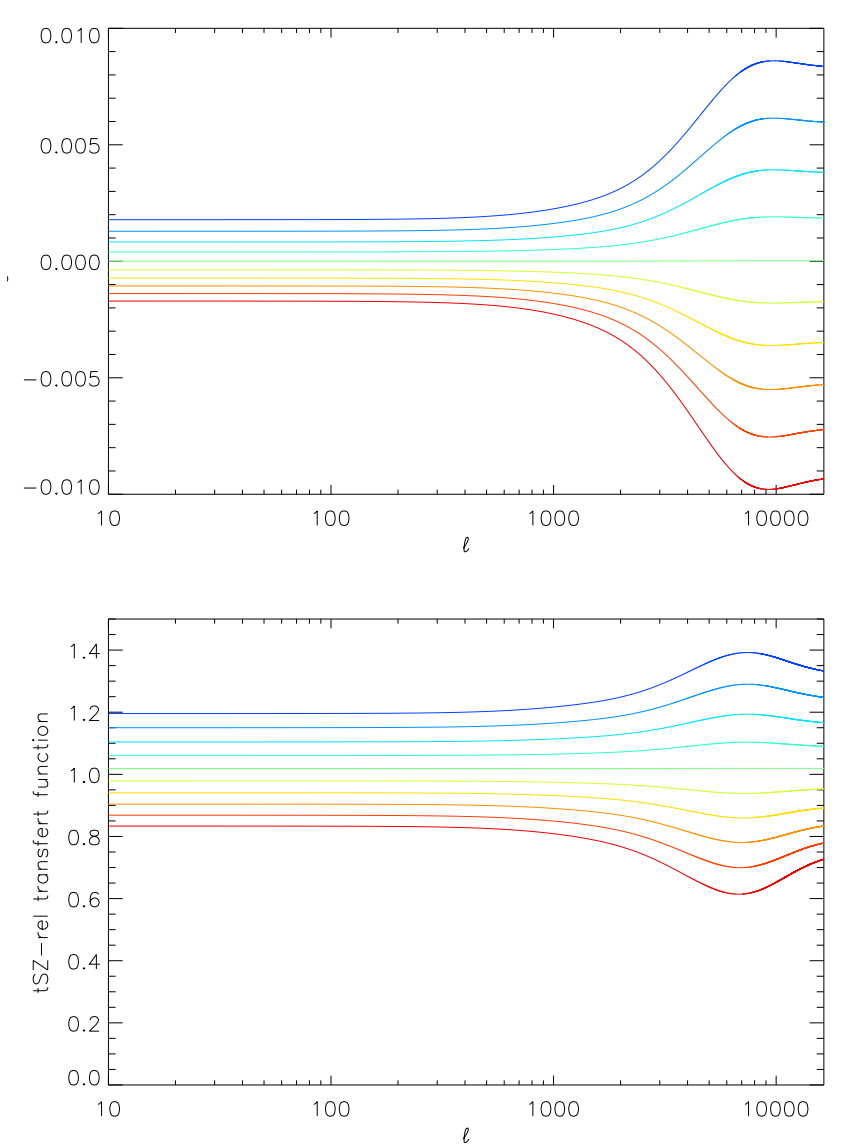

Fig. 8. Top panel: scale-dependant tSZ relativistic correction contamination into the tSZ $y$-map. Bottom panel: scale-dependant bias on tSZ relativistic correction amplitude due to our first-order linear approximation of the tSZ SED. Color ranging from blue to red indicates the considered temperature from 0 to $10 \mathrm{keV}$.

\subsection{Thermal dust emission}

The contribution of the infrared component in the MILCA $y$-map and $r$-map is crucial for tSZ relativistic correction measurement. If the contributions from galactic thermal dust and high- $z$ CIB are naturally reduced by the variance minimisation of the recovered maps, the contribution of the infrared emission produced by galaxies inside the clusters (see Planck Collaboration XXIII 2016, for a detailed analysis) is more problematic, and is significant even for intermediate-redshift galaxy clusters. By construction, this emission is spatially correlated with the tSZ effect. Such spatial correlation between astrophysical components leads to significant bias when blindly applying a minimum variance estimator, considering that the infrared signal can be used to remove the tSZ signal and thus reduce the variance in the recovered maps. Consequently, assumptions have to be made concerning the SED of the correlated thermal dust emission. These assumptions can be added in two ways in the MILCA method depending on the reliability of the thermal dust SED estimation: (i) Hard constraints into the SED matrix, $\mathcal{F}$, or (ii) soft constraints by modifying the channel covariance matrix $C_{\mathbf{T}}$ (see Hurier et al. 2013, for more details). For example, in a COrE+ like experiment, the highest frequency channels from 600 to $800 \mathrm{GHz}$ can be used to gain an estimation of the thermal dust SED. However, this estimation will have uncertainties that will translate into bias in the tSZ $y$-map and $r$-map. We estimated this bias using Eq. (17). We assumed a modified black body SED for the thermal dust with a temperature $T_{\mathrm{d}}=20 \mathrm{~K}$ and
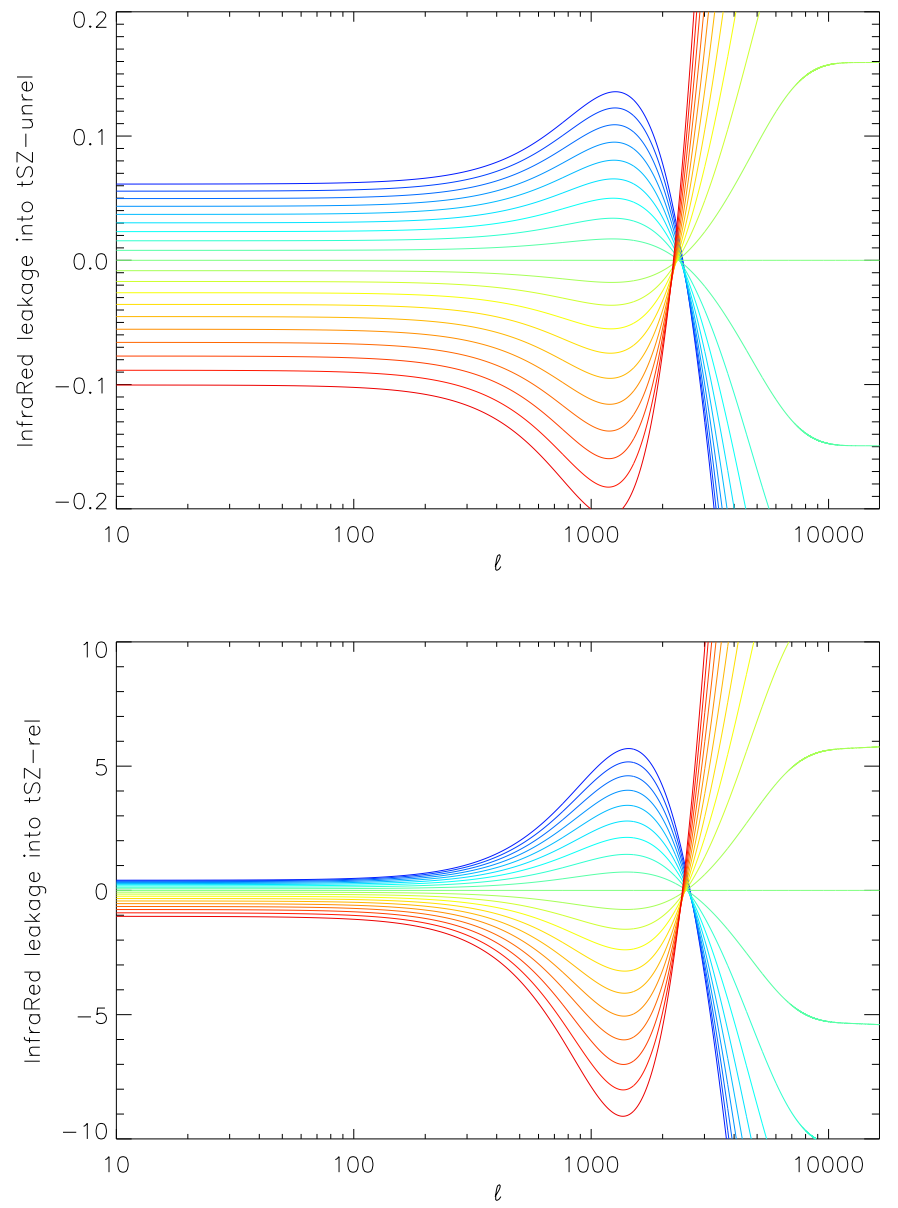

Fig. 9. Infrared component residuals into tSZ $y$-map (top panel) and $r$ map (bottom panel) as a function of the scale $\ell$ for a miss-estimation of infrared emission spectral properties. Color ranging from blue to red indicates the considered error on the thermal dust emission gray body spectral index, $\beta_{\mathrm{d}}$, from -0.2 to 0.2 . An intensity of $1 K_{\mathrm{CMB}}$ at $195 \mathrm{GHz}$ is used as a reference for the infrared emission.

a spectral index $\beta_{\mathrm{d}}=1.7$. These values provide a fair description of the local Universe infrared emission from galaxy clusters (Planck Collaboration XXIII 2016).

We have seen that the weights of the linear combination are strongly varying with the considered angular scale. Especially at small angular scale, the weights of the high frequency become more important (due to their higher resolution). Figure 9 presents the expected thermal dust leakage as a function of the angular scale and of the error on the assumed thermal dust emission gray body spectral index for a $1 K_{\mathrm{CMB}}$ source at $195 \mathrm{GHz}$. At such frequencies, the amplitude of the IR emission toward galaxy clusters is $\simeq 0.01-0.02$ Jy (Planck Collaboration XXIII 2016). This leads, for a beam of 7 arcmin FWHM, to an intensity at the peak of $10^{-6} \mathrm{~K}_{\mathrm{CMB}}$ for the IR emission. Thus from Fig. 9 we can deduce that an error of 0.2 on $\beta_{d}$ would induce a bias with an amplitude of $10^{-7}$ in the $y$-map and $10^{-6} \mathrm{keV}$ in the $r$-map.

Below $\ell=2000$, the amplitude of the leakage is small both in the tSZ $y$-map and $r$-map, and can safely be neglected. However, at higher- $\ell$, where only high frequency remains for the component separation, the bias strongly increases (we clarify that the curves at high- $\ell$ in Fig. 9 are going off-scale by one order of magnitude). This challenges the possibility to recover the high- $\ell$ part of the non-relativistic tSZ effect and tSZ relativistic correction at small angular scales as infrared contamination becomes a threat for a COrE+ like experiment. This 
statement also challenges low-mass galaxy cluster detection. Indeed, low-mass galaxy clusters are the most affected by infrared contamination, and require high angular resolution to be detected. Indeed, the $I_{\mathrm{IR}} / Y$ ratio scales as $M^{-0.7}$, as the IR flux, $I_{\mathrm{IR}}$, scales as $M$ (Planck Collaboration XI 2011), and $Y$ scales as $M^{5 / 3}$ (Planck Collaboration XI 2011; Sifón et al. 2013; Saliwanchik et al. 2015). The same arguments apply to highredshift galaxy clusters. This will probably limit the resolution at which the tSZ effect can be extracted, $\simeq 4$ arcmin FWHM.

Spatially varying thermal dust SED will have a similar effect on the reconstructed maps. Indeed, only one main thermal dust SED is cancelled by the linear combination. Consequently, if the thermal dust varies across the skies this will add a bias proportional to the mismatch between the main thermal SED and the contaminating SED. We stress that the localisation process of MILCA (spatial and angular scale localisation) aim at minimizing such an effect.

\subsection{Radio emission}

Numerous galaxy clusters host a bright radio-loud AGN at their center; well-known nearby galaxy clusters in this situation are Perseus and Virgo. Similarly to the infrared emission from member galaxies, the radio emission from AGNs will induce bias in the galaxy cluster tSZ signal reconstruction. This issue has been addressed since the tSZ reconstruction for the Planck experiment (Hurier et al. 2013). Contrary to the infrared emission, significant radio emission from clusters is produced by a single galaxy in the cluster. Consequently the radio emission from galaxy clusters, at least for resolved ones, can be separated from the tSZ effect using morphological criteria to measure the radio source SED properties. We model the radio galaxy SED as a power law $v^{\alpha_{r}}$, with $\alpha_{r}=0.5$ in intensity units (Planck Collaboration XIII 2011).

Figure 10 presents the leakage of a radio source into the tSZ $y$-map and $r$-map as a function of the angular scale and the error on the spectral index of the radio source. We observe that the bias is higher at low- $\ell$ where the lowest-frequencies contribute the most to the reconstruction of $\mathrm{tSZ}$ maps. For a bright radio source of $1 \mathrm{Jy}$ at $1.4 \mathrm{GHz}$, the expected peak intensity with a 7 arcmin FWHM beam is $10^{-5} \mathrm{~K}_{\mathrm{CMB}}$ at $195 \mathrm{GHz}$. This implies that above $\ell=1000$ for 0.2 uncertainties on $\alpha_{\mathrm{r}}$, the $y$-map will be contaminated up to $10^{-6}$ and the $r$-map up to $10^{-5} \mathrm{keV}$. Similarly to the infrared contamination, we observe that the tSZ $y$-map presents a small level of contamination that will at most correspond to $\simeq 1 \%$ of the tSZ Compton parameter flux. The tSZ $r$-map is more significantly affected up to a few percent below $\ell \simeq 1000$. Thus, tSZ relativistic correction extraction will require a careful AGN contamination cleaning to avoid significant bias.

Combining the information from tSZ maps mixing, infrared, and radio contamination we can identify that signal for $\ell$ below 2500 will enable a low-contamination, low-bias measurement of the tSZ relativistic correction for future experiments.

\section{Conclusion}

We have presented a new approach to extract tSZ-relativistic corrections using a component-separation method, MILCA. This approach performed the reconstruction of the tSZ Compton parameter and relativistic corrections through a linear combination of multi-frequency datasets. This allows us to provide robust estimations of the instrumental noise level and astrophysical component residuals (such as infra-red emission from member galaxies and radio-loud AGN emission from central galaxies).
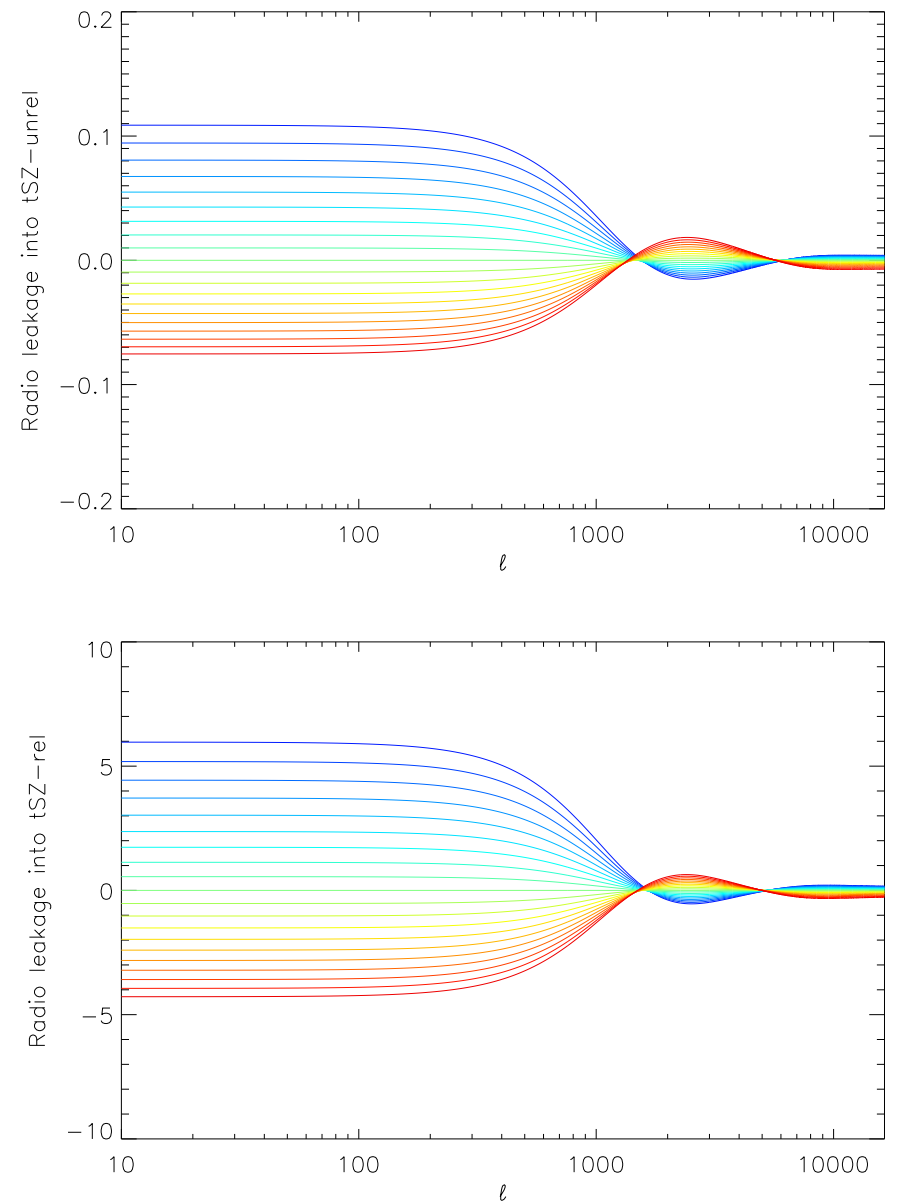

Fig. 10. Radio component residuals in the tSZ y-map (top panel) and $r$-map (bottom panel) as a function of the scale $\ell$ for a miss-estimation of radio emission spectral properties. Color ranging from blue to red indicates the considered error on the radio source SED spectral index, $\alpha_{\mathrm{r}}$, from -0.2 to 0.2 . An intensity of $1 K_{\mathrm{CMB}}$ at $195 \mathrm{GHz}$ is used as a reference for the radio emission.

We have characterized the MILCA transfer function to the tSZ relativistic corrections signal and performed a detailed analysis of the key frequency channels for both tSZ $y$-map and $r$-map reconstruction as a function of the angular scale for a COrE+ like experiment.

Our understanding of the foregrounds will be key to measuring the relativistic correction to the tSZ spectral distortion at high accuracy and high angular resolution. We identify that the tSZ $y$-map and $r$-map can be accurately recovered with a low level of bias up to $\ell \simeq 3000$, for ICM temperatures ranging from 0 to $15 \mathrm{keV}$.

Additionally, we have shown that relativistic corrections cannot be neglected for future high-sensitivity CMB experiments such as $\mathrm{COrE}+$, as their impact on pressure measurement, tSZ scaling relation, and tSZ power spectrum will be significant even for Compton parameter focussed analyses. This bias is particularly important when comparisons are made between tSZ data and numerical simulations.

We conclude that future high-resolution experiments will offer a unique window on the $\mathrm{tSZ}$ effect spectral distortion that will enable the possibility to perform detailed analysis of the ICM properties. Such additional probes will be complementary with X-ray studies of galaxy cluster properties (Tchernin \& Hurier, in prep.). 


\section{References}

Arnaud, M., Pratt, G. W., Piffaretti, R., et al. 2010, A\&A, 517, A92 Birkinshaw, M. 1999, Phys. Rep., 310, 97

Bleem, L. E., Stalder, B., de Haan, T., et al. 2015, ApJS, 216, 27

Bobin, J., Moudden, Y., Starck, J.-L., Fadili, J., \& Aghanim, N. 2008, Statistical Methodology, 5, 307

Carlstrom, J. E., Holder, G. P., \& Reese, E. D. 2002, ARA\&A, 40, 643

Ensslin, T. A., \& Hansen, S. H. 2004, ArXiv e-prints [astro-ph: 0401337]

Horowitz, B., \& Seljak, U. 2017, MNRAS, 469, 394

Hurier, G., Aghanim, N., Douspis, M., \& Pointecouteau, E. 2014, A\&A, 561, A143

Hurier, G., Douspis, M., Aghanim, N., et al. 2015, A\&A, 576, A90

Hurier, G., Hildebrandt, S. R., \& Macias-Perez, J. F. 2013, A\&A, 558, A118

Hurier, G. 2017, A\&A, 604, A93

Marriage, T. A., Acquaviva, V., Ade, P. A. R., et al. 2011, ApJ, 737, 61

Nozawa, S., Itoh, N., Kawana, Y., \& Kohyama, Y. 2000, ApJ, 536, 31

Planck Collaboration XI. 2011, A\&A, 536, A11
Planck Collaboration XIII. 2011, A\&A, 536, A13

Planck Collaboration I. 2014, A\&A, 571, A1

Planck Collaboration XXI. 2014, A\&A, 571, A21

Planck Collaboration XXIX. 2014, A\&A, 571, A29

Planck Collaboration XXII. 2016, A\&A, 594, A22

Planck Collaboration XXIII. 2016, A\&A, 594, A23

Planck Collaboration XXVII. 2016, A\&A, 594, A27

Planck Collaboration Int. V. 2013, A\&A, 550, A131

Pointecouteau, E., Giard, M., \& Barret, D. 1998, A\&A, 336, 44

Remazeilles, M., Delabrouille, J., \& Cardoso, J.-F. 2011, MNRAS, 410, 2481

Saliwanchik, B. R., Montroy, T. E., Aird, K. A., et al. 2015, ApJ, 799, 137

Sifón, C., Menanteau, F., Hasselfield, M., et al. 2013, ApJ, 772, 25

Sunyaev, R. A., \& Zeldovich, Y. B. 1972, Comments on Astrophysics and Space Physics, 4, 173

Wright, E. L. 1979, ApJ, 232, 348

Zemcov, M., Aguirre, J., Bock, J., et al. 2012, ApJ, 749, 114

Zemcov, M., Rex, M., Rawle, T. D., et al. 2010, A\&A, 518, L16 